\title{
Utility of the Neuropathic Pain Symptom Inventory in people with spinal cord injury
}

\author{
Marlon L. Wong $\mathbb{B}^{1,2} \cdot$ Loriann Fleming $^{1} \cdot$ Linda E. Robayo $^{1} \cdot$ Eva Widerström-Noga'
}

Received: 9 June 2019 / Revised: 22 July 2019 / Accepted: 23 July 2019 / Published online: 20 August 2019

(c) The Author(s), under exclusive licence to International Spinal Cord Society 2019

\begin{abstract}
Study design Cohort/psychometric study

Objectives The primary objective was to determine the psychometric properties and the utility of the Neuropathic Pain Symptom Inventory (NPSI) in subgrouping people with moderate to severe neuropathic pain after spinal cord injury (SCI). Setting University-based laboratory in Miami, FL USA.

Methods Seventy-two people with chronic SCI and neuropathic pain were included in this study. The NPSI, Numeric Rating Scale (NRS), Multidimensional Pain Inventory pain severity and perceived support subscales (MPI-PS and MPI-S, respectively), and the Coping Strategies Questionnaire were administered. The NPSI was administered twice, with a 2-4week period between measurement sessions.

Results The NPSI total score demonstrated good internal consistency with a Cronbach's alpha of 0.70. The test-retest reliability (intraclass correlations) ranged from 0.65 to 0.73 for the NPSI subscores and 0.79 for the total NPSI score. Further, construct validity was supported by moderate and significant positive correlations with the pain intensity NRS and pain severity subscale of the MPI (MPI-PS) $(r>0.40)$. Cluster analysis of factor scores derived from NPSI subscales, NRS, and MPI-PS scores revealed three distinct subgroups: (1) low-moderate, (2) moderate, and (3) high pain symptom severity with mean NPSI sum scores of 7.1, 17.5, and 33.8, respectively.

Conclusion The NPSI demonstrated good psychometric properties in people with neuropathic pain after SCI. Moreover, it has utility for establishing pain symptom phenotypes.
\end{abstract}

\section{Introduction}

Neuropathic pain is an underrecognized contributing factor to the burden and diminished quality of life for people with spinal cord injury (SCI) [1]. In fact, neuropathic pain affects a large proportion of people with SCI, with an estimated

Supplementary information The online version of this article (https:// doi.org/10.1038/s41393-019-0338-5) contains supplementary material, which is available to authorized users.

Marlon L. Wong

mwong2@miami.edu

1 Miami Project to Cure Paralysis and Department of Neurological Surgery, Miller School of Medicine, University of Miami, Miami, FL, USA

2 Department of Physical Therapy, Miller School of Medicine, University of Miami, Miami, FL, USA prevalence rate of 53\% [2]. Because neuropathic pain is known to be more severe and resistant to treatment than other types of pain [3], detailed diagnosis and effective management of neuropathic pain after SCI is an important component of medical care and research aimed at optimizing the quality of life in this population. However, currently there are no interventions that provide consistent and widespread benefit for neuropathic pain after SCI [4]. Thus, detailed diagnosis and characterization of neuropathic pain associated with SCI is required for developing more personalized and effective pain management $[4,5]$.

The Neuropathic Pain Symptom Inventory (NPSI) is one of the most widely used tools for characterizing neuropathic pain symptom severity, and it has been validated in over 50 different languages $[6,7]$. The NPSI is comprised of five subscales, each representing different dimensions of neuropathic pain: burning spontaneous pain (burning), pressing spontaneous pain (pressing), paroxysmal pain (paroxysmal), evoked pain (evoked), and paresthesia/dysesthesia. This factorial structure makes the NPSI well suited for 
investigating the relationships between symptomology, treatment responses, and ultimately the underlying physiological mechanisms of neuropathic pain $[8,9]$. Moreover, the NPSI has good construct validity, high test-retest reliability, and good sensitivity to change in people with peripherally mediated neuropathic pain $[6,10-15]$. Therefore, the NPSI may also be a valuable tool for characterizing neuropathic pain symptom severity in people with SCI neuropathic pain.

Although this measure has been used in SCI chronic pain populations [16], the psychometric properties of the NPSI have not been established for the SCI neuropathic pain population. The derivation study for the NPSI used a cohort of people with heterogeneous etiologies of neuropathic pain, of which only $8 \%$ had SCI $(n=14)$ [6]. Further, no analysis was conducted to determine if the psychometric properties of the NPSI differed between groups based on etiology of neuropathic pain. Nagoshi et al. [16] used the NPSI to characterize neuropathic pain in 72 patients with SCI, providing important epidemiological information on the severity of specific neuropathic pain symptoms and the relationship of these symptoms with quality of life. However, they did not assess the psychometric properties of the NPSI.

Our primary objective was to fill this gap by determining the psychometric properties of the NPSI in people with neuropathic pain after SCI. In addition, our goal was to determine the utility of the NPSI for subgrouping people based on pain symptom severity across multiple instruments. Improving our ability to establish clinically meaningful subgroups is an ongoing challenge and focus across the field of pain research, and pain symptom severity is an important consideration for multidimensional subgrouping. Moreover, improving the understanding of multidimensional SCI neuropathic subgroups may optimize pain management for people with neuropathic pain after SCI by allowing for tailored treatment approaches [5].

\section{Methods}

\section{Participants}

This study was based on a subset of data from a larger study involving people with SCI and neuropathic pain [17]. Seventy-two consecutive people with a history of traumatic SCI (occurring at least 1 year before study enrollment), and with neuropathic pain of at least moderate intensity (pain severity $\geq 4$ on the $0-10$ Numeric Rating Scale [NRS]), were recruited through the University of Miami between November 23, 2010 and October 29, 2012. The presence of neuropathic pain was determined according to location, description, and apparent origin using the criterion described by Siddall et al. [18]. Participants were at least 18 years old, and able to speak and understand English. Participants were excluded if they had cognitive impairment (score of $<20$ on the Mini-Mental State Exam), major depression, current (within a year) drug abuse, or significant chronic health conditions other than SCI.

\section{Instruments}

\section{Numeric Rating Scale}

An 11-point NRS was used to assess average neuropathic pain severity over the last week. The scale ranged from 0 to 10 , with 0 representing "no pain" and 10 representing "the most intense pain imaginable". The NRS demonstrates good reliability and validity across a wide range of painful conditions, including chronic neuropathic pain [19]. Several review articles identify the NRS as the most appropriate response scale for the assessment of pain in adults, and it is recommended as the gold standard by the IMMPACT guidelines for pain measurement in clinical trials and the National Institutes of Health Toolbox pain assessment [19-21].

\section{Multidimensional Pain Inventory: SCI version (MPI-SCl)}

The West Haven-Yale MPI [22] is a comprehensive instrument designed to assess a range of self-reported behavioral and psychosocial factors associated with chronic pain syndromes. Based on our previous research [23, 24], we determined that a modified version of the MPI, the MPI-SCI, was appropriate for use with people who experience pain associated with their SCI. The total scale consists of 50 items, but only the pain severity (MPI-PS) and perceived support (MPI-S) subscales were used to assess construct validity (convergent and divergent validity, respectively) of the NPSI. The MPI-PS and MPI-S each consist of three items, which are answered on a 7-point Likert scale. Subscale scores are the mean of all the three items with a potential range of 0-6. The MPI-PS asks participants to rate their current pain severity, average pain severity over the past week, and suffering associated with their pain. The MPI-S asks participants to rate how helpful, worried, and attentive their spouse or significant other is in relation to their pain.

\section{Coping Strategies Questionnaire (CSQ)}

Elevated pain catastrophizing is a risk factor for poorer outcomes with pain management [25]. However, catastrophizing is also modifiable and amenable to cognitive behavioral interventions; [26] thus, catastrophizing is an important factor to consider and a potential treatment target in people with neuropathic pain after SCI. The 6-item 
catastrophizing subscale of the CSQ [27] was used to assess catastrophizing in this study. Each item is rated on a 0-6 scale from "Never do" to "Always do that when in pain", with higher scores indicating more frequent catastrophizing in response to pain. The subscale score is the mean of all six items. The catastrophizing subscale has demonstrated excellent reliability, validity, and is significantly associated with measures of adjustment to various types of chronic pain including SCI [27, 28].

\section{Analyses}

\section{Internal consistency}

Cronbach's alpha test was used to analyze the internal consistency among NPSI subscales. Good internal consistency was defined as a Cronbach's alpha score above 0.60.

\section{Test-retest reliability}

The NPSI was administered twice, with a 2-4-week period between testing sessions, and two-way mixed, single measures intraclass correlation coefficient (ICC) estimates and their $95 \%$ confidence intervals were calculated to determine the test-retest reliability. Good reliability was defined as ICC values above 0.60 based on single measures, consistency, two-way mixed model.

\section{Construct validity}

For convergent validity, we used Pearson product correlation coefficient analysis to determine the relationship of the total NPSI (NPSI sum) and NPSI subscale scores with the NRS and the MPI-PS. A statistically significant Pearson product correlation coefficient value $>0.30$ is indicative of a moderate correlation, and demonstrates support of convergent validity. Divergent validity was determined by analyzing the correlation between NPSI sum scores and the MPI-S as this scale was not expected to strongly correlate to NPSI scores.

\section{Pain severity subgroups}

All NPSI subscale scores, the NRS, and the MPI-PS scores were entered into an exploratory factor analysis. This statistical approach may be used for determining the relationships among multiple factors and for explaining these variables in terms of their common underlying dimensions or factors. A principal component analysis based on a correlation matrix was used for extracting factors and producing factor scores. Bartlett's test of sphericity was considered adequate with high $\chi^{2}$ and probability lower than $5 \%$. The Kaiser-Meyer-Olkin measure for sampling adequacy was also calculated; a coefficient higher than 0.60 was considered adequate. The Kaiser-Meyer-Olkin measure for sampling adequacy was 0.76 and the Bartlett's test of sphericity was $145.2(p=0.00)$, demonstrating that the cohort data were suitable for factor analysis.

A two-step cluster procedure based on the previously obtained factor scores was used to define homogeneous subgroups or "clusters" inherent in the data from the 72 participants with SCI and neuropathic pain. In the SPSS 24 two-step clustering procedure, cases are initially assigned to preclusters based on automatic preset algorithms using a sequential clustering approach. This algorithm automatically scans the records one by one and determines if the current record should merge with a previously formed precluster or start a new precluster based on the assignment that maximizes the log-likelihood as the distance measure. The number of clusters was determined by the automatic IBM SPSS 24 for Windows default criterion (Schwarz's criterion). Once the number of clusters was determined, we compared the clusters with respect to the variables included in the cluster analysis to determine the appropriateness of the clusters. For external validation purposes, one-way ANOVA analyses were used to compare the clusters with regard to demographic and injury-related factors, MPI subscores, and the catastrophizing subscale of the Coping Strategies Questionnaire; Tukey HSD correction was used for post hoc group comparisons. To determine which of the five NPSI subscales that best predicted cluster membership, we conducted a stepwise discriminant analysis. In order to meet the assumptions of the analysis we examined data for normal distribution and homoscedasticity, excluded variables with significant intercollinearity ( 0.7 or above), and used Box's $\mathrm{M}$ to determine equality in covariance matrices among subgroups. We used the probability of $\mathrm{F}$ to select variables into the model and Wilk's lambda to determine which variables contributed significantly to the discriminant analysis. At each step, the variable that minimized the overall Wilks' lambda was entered. Analysis of covariance was used to assess for potential confounding effects of intergroup differences in demographic factors.

\section{Results}

Seventy-two participants were enrolled in the study and completed data collection for visit A, of which sixty-seven also returned for visit B. Visit B values were only used to determine test-retest reliability for the NPSI. All other analyses were calculated using Visit A values. Mean scores for pain intensity NRS (range 0-10) and MPI-PS (range $0-6)$ were $6.6 \pm 1.8$ and $3.5 \pm 1.3$, respectively. Demographic, pain, medication, and injury information for the cohort can be found in Table 1 . 
Table 1 Participant background information

\begin{tabular}{|c|c|c|c|}
\hline & $\begin{array}{l}\text { LMNP } \\
N=23\end{array}$ & $\begin{array}{l}\text { HMNP } \\
N=37\end{array}$ & $\begin{array}{l}\mathrm{SNP} \\
N=12\end{array}$ \\
\hline \multicolumn{4}{|l|}{$\operatorname{Sex}^{\mathrm{a}}(\%)$} \\
\hline Men & $22(95.7)$ & $26(70.3)$ & $11(91.7)$ \\
\hline Women & $1(4.3)$ & $11(29.7)$ & $1(8.3)$ \\
\hline Age $(\text { years })^{\text {ns }}$ & $35.8 \pm 14.7$ & $38.5 \pm 10.7$ & $35.53 \pm 9.7$ \\
\hline Years with $\mathrm{SCI}^{\mathrm{ns}}$ & $9.7 \pm 8.1$ & $12.1 \pm 10.2$ & $10.9 \pm 9.0$ \\
\hline $\begin{array}{l}\text { Multidimensional Pain } \\
\text { inventory_pain severity } \\
\text { subscale }(0-6)\end{array}$ & $2.3 \pm 1.1$ & $3.9 \pm 0.9$ & $4.8 \pm 0.7$ \\
\hline $\begin{array}{l}\text { Numerical Pain Rating } \\
\text { Scale of worst neuropathic } \\
\text { pain }(0-10)\end{array}$ & $5.5 \pm 1.3$ & $6.8 \pm 1.7$ & $8.0 \pm 1.4$ \\
\hline \multicolumn{4}{|c|}{ Worst neuropathic pain distribution (\%) } \\
\hline Pain at-level of injury & $5(21.7)$ & $12(32.4)$ & $4(33.3)$ \\
\hline Pain below-level of injury & $18(78.3)$ & $25(67.6)$ & $8(66.7)$ \\
\hline $\begin{array}{l}\% \text { with complete } \\
\text { impairment (AIS-A) }\end{array}$ & $13(56.5)$ & $22(59.5)$ & $7(58.3)$ \\
\hline Cervical SCI level (\%) & $15(65.2)$ & $18(48.6)$ & $5(41.7)$ \\
\hline $\begin{array}{l}\text { Below cervical SCI } \\
\text { level }(\%)\end{array}$ & $8(34.8)$ & $19(51.4)$ & $7(58.3)$ \\
\hline \multicolumn{4}{|l|}{ Medication use (\%) } \\
\hline Antispasticity & $11(47.8)$ & $14(37.8)$ & $4(33.3)$ \\
\hline Anti-inflammatory & $7(30.4)$ & $9(24.3)$ & $2(16.7)$ \\
\hline Anticonvulsant & $6(26.1)$ & 8 (21.6) & $2(16.7)$ \\
\hline Opioid & $3(13.0)$ & $12(32.4)$ & $6(50.0)$ \\
\hline $\begin{array}{l}\text { Muscle relaxant or } \\
\text { sedatives }\end{array}$ & $10(43.5)$ & $13(35.1)$ & $5(41.7)$ \\
\hline Antidepressants & $2(8.7)$ & $2(5.4)$ & $3(25.0)$ \\
\hline
\end{tabular}

${ }^{a}$ significant group differences (Kruskal-Wallis Test $\chi^{2}(2)=6.997$, $p=0.03$ )

$n s$ no significant differences between groups, $L M N P$ low-moderate neuropathic pain, $H M N P$ high-moderate neuropathic pain, $S N P$ severe neuropathic pain, AIS-A American Spinal Injury Association Impairment Scale A

\section{Psychometric properties of the NPSI in people with moderate to severe neuropathic pain after $\mathrm{SCl}$}

The NPSI sum score demonstrated good test-retest reliability, with an ICC of 0.79 . Further, most of the subscales demonstrated good test-retest reliability with ICC values for burning, pressing, paroxysmal, evoked, and paresthesial dysesthesia of $0.65,0.72,0.66,0.68$, and 0.73 , respectively (Table 2). The internal consistency of the NPSI total score was also good, with a Cronbach's alpha value of 0.7. Thus, the NPSI appears to have good stability and reliability in people with SCI.

The NPSI also demonstrated good construct validity. Convergent validity was demonstrated through moderate and significant correlations of the NPSI sum score with NRS $(r=0.47, p=0.001)$ and MPI-PS scores $(r=0.59$, $p=0.001$ ) (Table 2). Out of the NPSI subscales evoked pain was the only NPSI subscale that was neither significantly correlated to NRS MPI-PS, and the paroxysmal subscale was weakly but significantly correlated to the MPI-PS and to the NRS. All other NPSI subscales were moderately and significantly correlated to both NRS and MPI-PS scores (Table 2). Divergent validity was demonstrated through the absence of any significant correlations between the NPSI and the MPI-S (Table 2). Thus, the NPSI sum score, and most of the subscale scores, appeared to correspond with the pain severity scores of other instruments. However, the evoked pain subscale may reflect a neuropathic symptom that is not well captured by NRS or MPI-PS.

\section{NPSI scores in people with neuropathic pain after SCI}

The mean scores for NPSI sum and the subscales (burning, pressing, paroxysmal, evoked, paresthesia/dysesthesia) were $16.9 \pm 10.0,4.7 \pm 3.4,3.0 \pm 3.0,3.0 \pm 3.1,1.8 \pm 2.1$, and $4.3 \pm 3.0$, respectively (Fig. 1). Paresthesia/dysesthesia was the most common symptom reported (83\% of participants), followed by burning then pressing (75\% and $69 \%$ of participants, respectively). Paroxysmal and evoked pain were the least commonly reported symptoms, with $64 \%$ and $60 \%$ of participants reporting these symptoms, respectively.

\section{Pain symptom severity subgroups}

The factor analysis, based on the NPSI subscales, pain intensity, and the MPI-PS subscale scores, resulted in two factors (Table 3), and the resulting factor scores were used in a two-step cluster analysis to determine neuropathic symptom severity subgroups in the sample. The cluster analysis revealed three distinct groups: (1) low-moderate neuropathic pain (LMNP; $n=23$ ), (2) high-moderate neuropathic pain (HMNP; $n=37$ ), and (3) severe neuropathic pain (SNP; $n=$ 12) pain groups. The NPSI sum scores for these groups were 7.1, 17.5, and 33.8, respectively. Cluster differences in NPSI subscale and sum scores, NRS, and MPI-PS scores are found in Fig. 2. A stepwise discriminant analysis was then conducted to predict which combination of the five NPSI subscales best classified cluster membership. The, pressing, burning, paroxysmal and paresthesia/dysesthesia subscales (Wilks' lambda: 0.296, 0.238, 0.184, and 0.180, respectively) correctly and significantly $(p<0.001)$ classified cluster membership in $90.3 \%$ of the cases (Table 4 ).

\section{Comparisons among neuropathic pain symptom severity subgroups}

The groups did not differ in age $(p=0.72)$, age at time of SCI $(p=0.97)$, or chronicity of SCI $(p=0.69)$. The groups 
Table 2 NPSI psychometric properties in people with moderate to severe neuropathic pain after SCI

\begin{tabular}{|c|c|c|c|c|}
\hline $\begin{array}{l}\text { Subscale (number or } \\
\text { items) } 0 \text { - } 10 \text { score range } \\
\text { for each }\end{array}$ & $\begin{array}{l}\text { Test-retest ICC } r \\
\text { value }(95 \% \\
\text { confidence interval) }\end{array}$ & $\begin{array}{l}\text { Correlation to } \\
\text { NRS } r \text { value } \\
(p \text { value })\end{array}$ & $\begin{array}{l}\text { Correlation to } \\
\text { MPI-PS } r \text { value } \\
\text { ( } p \text { value })\end{array}$ & $\begin{array}{l}\text { Correlation to } \\
\text { MPI-S } r \text { value } \\
(p \text { value })\end{array}$ \\
\hline Burning (1) & $0.65(0.49-0.77)$ & $0.45(0.001)$ & $0.49(0.001)$ & $-0.10(0.408)$ \\
\hline Pressing (2) & $0.72(0.58-0.82)$ & $0.35(0.003)$ & $0.43(0.001)$ & $-00.8(0.949)$ \\
\hline Paroxysmal (2) & $0.66(0.50-0.78)$ & $0.24(0.047)$ & $0.30(0.010)$ & $0.01(0.939)$ \\
\hline Evoked pain (3) & $0.68(0.52-0.79)$ & $0.12(0.338)$ & $0.22(0.064)$ & $0.02(0.902)$ \\
\hline $\begin{array}{l}\text { Paresthesia/ } \\
\text { dysesthesia (2) }\end{array}$ & $0.73(0.60-0.83)$ & $0.39(0.001)$ & $0.50(0.001)$ & $0.12(0.328)$ \\
\hline Total NPSI score (10) & $0.79(0.68-0.86)$ & $0.47(0.001)$ & $0.59(0.001)$ & $0.01(0.955)$ \\
\hline
\end{tabular}

ICC intraclass correlation coefficient, NRS Numeric Rating Scale, MPI-PS Multidimensional Pain Inventory pain severity subscale, MPI-S Multidimensional Pain Inventory spousal support subscale, NPSI Neuropathic Pain Symptom Inventory

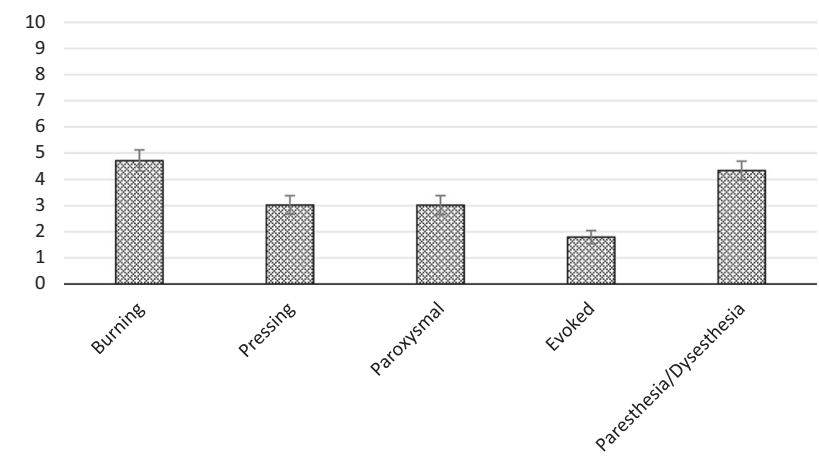

Fig. 1 Mean NPSI Subscale Scores for Visit A

Table 3 Pattern matrix from factor analysis

\begin{tabular}{lcc}
\hline Variable & Factor 1 & Factor 2 \\
\hline NRS & 0.89 & -0.15 \\
MPI-PS & 0.83 & \\
NPSI-burning & 0.76 & \\
NPSI-parysthesia/dysethesia & 0.68 & 0.13 \\
NPSI-pressing & & 0.81 \\
NPSI-paroxysmal & & 0.75 \\
NPSI-evoked & -0.12 & 0.63 \\
\hline
\end{tabular}

NRS Numeric Rating Scale, MPI-PS Multidimensional Pain Inventory pain severity subscale, NPSI Neuropathic Pain Symptom Inventory

did differ in sex $(p=0.03)$, with only one female in the LMNP and SNP groups and eleven females in the HMNP group. However, sex did not have a main effect on the NPSI subscale or sum scores, the MPI-PS, nor on the catastrophizing subscale of the Coping Strategies Questionnaire ( $p$ values ranging from 0.22 to 0.92 ). The SNP subgroup demonstrated significantly higher catastrophizing compared with the LMNP subgroup $(p=0.05)$ with mean scores of 10.4 and 4.3, respectively, on the catastrophizing subscale of the Coping Strategies Questionnaire (Fig. 3).

\section{Discussion}

This study is the first to provide a description of the psychometric properties of the NSPI in a moderately sized cohort of people with moderate to severe neuropathic pain after SCI. Previous studies investigating the psychometric properties of the NPSI used cohorts with heterogeneous etiologies of neuropathic pain, and included only a small percentage of participants with SCI. The percentage of participants with SCI typically ranged from 6 to $12 \%$ $[6,10-13]$, and in one study $18 \%(n=10)$ of participants had SCI [14]. Moreover, none of these studies analyzed differences in NPSI scores or psychometric properties between etiology-based groups. Therefore, it was unknown if the findings in these studies are representative of the psychometric properties of the NPSI in people with neuropathic pain after SCI, or if the NPSI performs differently in people with neuropathic pain after SCI compared with people with neuropathic pain of peripheral etiology. We only found one study that compared NPSI scores between participants with different etiologies for neuropathic pain [29], but they also had a small percentage of SCI participants $(6 \%)$, and they only reported on the prevalence of specific symptoms and the correlation of symptoms with quantitative sensory testing. Similarly, another study on NPSI scores in 72 participants with SCI [16] only reported on severity of specific symptoms and the correlation of symptoms with quality of life.

The NPSI demonstrated good psychometric properties in people with moderate to severe neuropathic pain after SCI. Although the NPSI instructs users to rate their symptoms over the last $24 \mathrm{~h}$, a relatively short period of time, the NPSI demonstrated surprisingly good test-retest reliability over a much longer period. This finding supports previous results of neuropathic pain following SCI as a chronic and relatively stable condition [30]. The moderate and significant correlation of the NPSI with the NRS and MPI-PS, and lack 
Fig. 2 Cluster differences in pain symptom severity. LMNP low-moderate neuropathic pain, HMNP high-moderate neuropathic pain, SNP severe neuropathic pain, NRS Numeric Rating Scale, MPI-PS Multidimensional Pain Inventory pain severity subscale, NPSI Neuropathic Pain Symptom Inventory

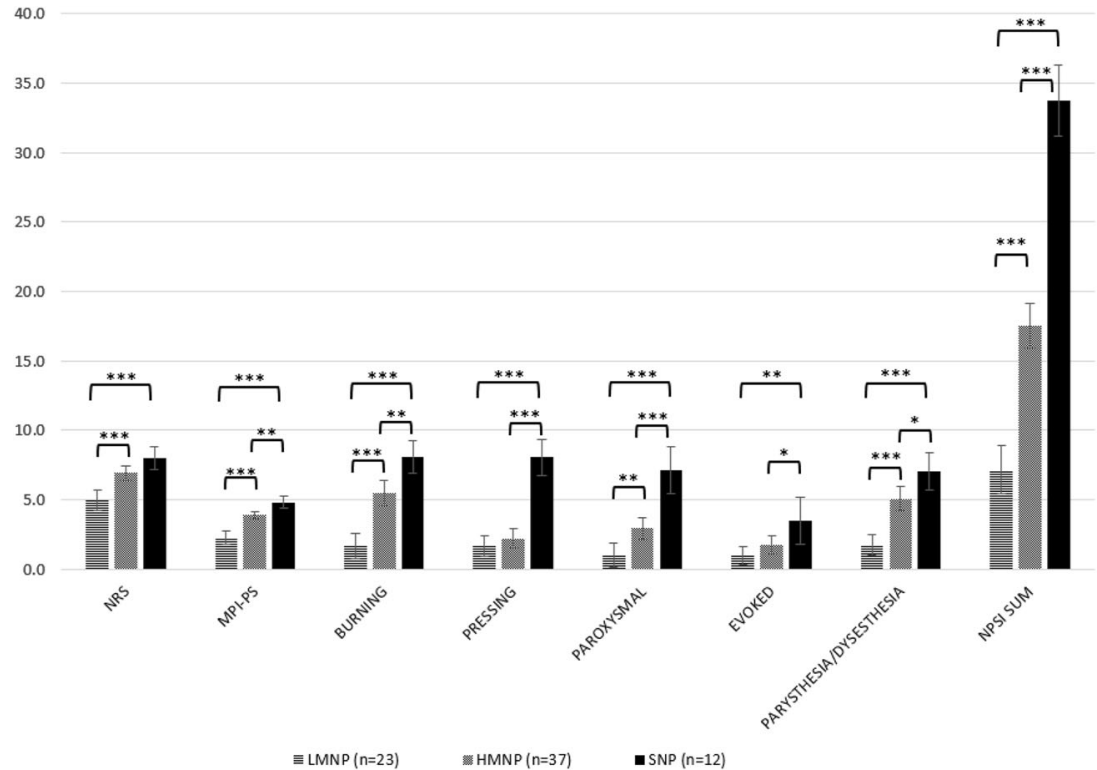

Table 4 Stepwise discriminate analysis classification results

\begin{tabular}{llll}
\hline \multirow{2}{*}{ Subgroup } & \multicolumn{2}{l}{ Predicted group membership } \\
\cline { 2 - 4 } & LMNP $(n, \%)$ & $\operatorname{HMNP}(n, \%)$ & $\operatorname{SNP}(n, \%)$ \\
\hline LMNP & $20,87.0$ & $3,13.0$ & $0,0.0$ \\
HMNP & $4,10.8$ & $33,89.2$ & $0,0.0$ \\
SNP & $0,0.0$ & $0,0.0$ & $12,100.0$ \\
\hline
\end{tabular}

LMNP low-moderate neuropathic pain, $H M N P$ high-moderate neuropathic pain, $S N P$ severe neuropathic pain

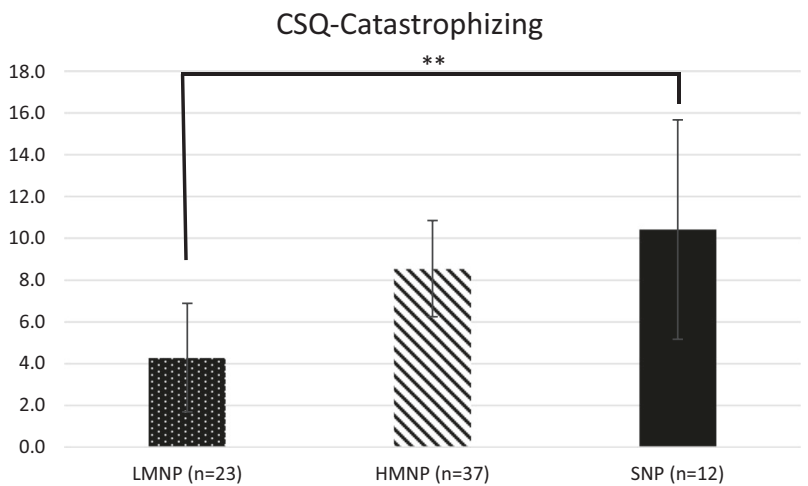

Fig. 3 Cluster differences in catastrophizing. CSQ Coping Strategies Questionnaire, LMNP low-moderate neuropathic pain, HMNP highmoderate neuropathic pain, SNP severe neuropathic pain

of significant correlation between the NPSI and MPI-S, support the construct validity for using the NPSI to assess severity of neuropathic pain symptoms in people with moderate to severe neuropathic pain after an SCI. The NPSI also demonstrated good internal consistency in this cohort, with a Cronbach's alpha value just slightly below those reported in studies of people with peripherally mediated neuropathic pain (range between 0.75 and 0.90) [11, 13, 15]. Although, the NPSI demonstrated good internal consistency, some of the subscales appear to better reflect overall neuropathic pain symptom severity than others in people with neuropathic pain after SCI. Specifically, the stepwise discriminant analysis showed that a combination of pressing, burning, paroxysmal, and paresthesia/dysesthesia subscales correctly predicted $90.3 \%$ of cluster membership. Moreover, SNP group membership was correctly classified in $100 \%$ of the cases. However, the evoked pain subscale did not significantly contribute to the subgroup classification and was also the least severe neuropathic pain symptom reported in this study. Overall, these findings establish the psychometric properties for, and support the utility of, the NPSI in people with neuropathic pain after SCI.

The good test-retest reliability for the whole NPSI scale observed in this study ( $\mathrm{ICC}=0.79$ ) was consistent with the findings reported in the development of the NPSI [6] (ICC $=0.88$ ) and other validation studies of the NPSI (ICC values ranging from 0.68 to 0.89 ) [11-14]. The test-retest reliability of the NPSI in people with SCI may be further enhanced by modifying the NPSI user instructions to reflect symptom intensity over the last week, rather than just the last $24 \mathrm{~h}$, in particular with respect to paroxysmal and evoked pain. In fact, a qualitative study on the cross-cultural adequacy of the NPSI found that some participants with neuropathic pain felt that the 24-h recall period was too short [31]. Using a 1-week recall period may be more clinically meaningful given that neuropathic pain tends to be persistent after an SCI. 
The positive correlations of the NPSI sum score with the pain intensity NRS $(r=0.40, p=0.00)$ and with the MPIPS $(r=0.56, p=0.00)$ scores confirmed the relevance of the NPSI to both pain intensity and severity. These findings also suggest that the NPSI provides additional and useful information that provide a better understanding of neuropathic pain severity in this population. Other studies that have compared NPSI and NRS scores used cohorts with heterogeneous etiologies of neuropathic pain and also found moderate and significant correlations, with $r$ values ranging between 0.40 and 0.60 [6, 12, 14]. Further analysis of the subscales revealed that all subscales shared similar moderate and significant relationships with the NRS and MPI-PS, except for the evoked pain subscale (Table 2).

It is possible that the overall perception and appraisal of neuropathic pain severity after SCI may be less influenced by evoked pain symptoms than other pain symptoms (i.e., spontaneous burning and pressing pain), and that NRS and MPI-PS ratings best reflect pain symptoms of a more constant nature rather than intermittent. This may be due to the fact that some people with SCI are well aware of factors that may trigger or exacerbate their pain and may therefore actively avoid these triggers (e.g., prolonged sitting, full bladder, cold stimuli). This idea is also supported by the fact that the evoked pain subscale did not contribute to the cluster classification. Nevertheless, the evoked pain subscale provides important information regarding the neuropathic pain symptoms that occur after an SCI. For example, the presence of evoked pain symptoms provides insight to the potential underlying mechanisms of a person's neuropathic pain, and therefore the optimal course of treatment. Further, the presence of evoked pain symptoms is a perceived barrier for those living with chronic pain after SCI [5].

Our study shows that the NPSI can complement general pain intensity and severity assessments to establish more detailed pain symptom severity subgroups. Numerous authors have commented on the importance of detailed pain symptom characterization for clinical and research purposes [5, 6, 12, 14]. However, taking a good pain history, and utilizing an array of validated instruments to adequately characterize pain symptoms, is time consuming and burdensome which limits compliance with this recommendation. We found that a combination of the NPSI subscales could correctly identify individuals with low-moderate, moderate, of high neuropathic pain symptom severity based on the groups defined by the NPSI subscales, the NRS, and the MPI-PS. Moreover, these groups appear to utilize different coping strategies with the SNP group engaging in more catastrophizing than the LMNP group. Thus, knowledge of pain symptom severity group membership may help to guide and select optimal treatment strategies. For example, cognitive behavioral therapy is known to decrease catastrophizing [26] and targeting this intervention to people in the high neuropathic pain symptom severity group may improve outcomes. However, this is speculative and additional research in this area is needed. In summary, the good psychometric properties and the ability of the NPSI to assess neuropathic pain symptom severity suggest that the NPSI is a useful instrument for general determination of neuropathic pain severity and for subgrouping of neuropathic pain symptoms associated with SCI.

\section{Limitations and future research}

This study only included people with moderate to severe neuropathic pain after SCI (NRS $\geq 4 / 10)$. Therefore, the NPSI may not be as useful in cases with mild neuropathic pain after SCI. Future studies should include individuals with mild neuropathic pain as well. Furthermore, longitudinal studies are needed to determine the clinical utility of the pain symptom severity subgroups identified in this study.

\section{Conclusion}

In conclusion, The NPSI demonstrated good psychometric properties in a cohort of people with neuropathic pain after SCI. The complete NPSI instrument and all individual subscales of the NPSI are useful for determining neuropathic pain symptom severity in the SCI neuropathic pain population and thus for neuropathic pain phenotyping. Further, the Pressing, Burning, Paroxysmal, and Paresthesia/Dysesthesia subscales may be particularly useful for determining an individual's appraisal of overall neuropathic pain severity after SCI.

\section{Data archiving}

All data generated or analyzed during this study are included in this published article and its supplementary information files.

Funding This study was funded by the Craig Neilsen Foundation.

Author contributions MLW was responsible for data analysis and writing the report. LF was responsible for data collection and also participated in writing the report. LER was responsible for data analysis and writing the report. As the senior author, EWN was responsible for designing the protocol, conducting the research, extracting the data, and writing the report.

\section{Compliance with ethical standards}

Conflict of interest The authors declare that they have no conflict of interest. 
Ethics We certify that all applicable institutional and governmental regulations concerning the ethical use of human volunteers were followed during the course of this research.

Publisher's note: Springer Nature remains neutral with regard to jurisdictional claims in published maps and institutional affiliations.

\section{References}

1. Widerstrom-Noga EG, Felipe-Cuervo E, Yezierski RP. Chronic pain after spinal injury: interference with sleep and daily activities. Arch Phys Med Rehabil. 2001;82:1571-7.

2. Burke D, Fullen BM, Stokes D, Lennon O. Neuropathic pain prevalence following spinal cord injury: a systematic review and meta-analysis. Eur J Pain. 2017;21:29-44.

3. Attal N, Cruccu G, Baron R, Haanpaa M, Hansson P, Jensen TS, et al. EFNS guidelines on the pharmacological treatment of neuropathic pain: 2010 revision. Eur J Neurol. 2010;17:1113-e88.

4. Widerstrom-Noga E. Neuropathic pain and spinal cord injury: phenotypes and pharmacological management. Drugs. 2017;77:967-84.

5. Widerstrom-Noga E, Anderson KD, Perez S, Martinez-Arizala A, Cambridge JM. Subgroup perspectives on chronic pain and its management after spinal cord injury. J Pain. 2018;19:1480-90.

6. Bouhassira D, Attal N, Fermanian J, Alchaar H, Gautron M, Masquelier E, et al. Development and validation of the Neuropathic Pain Symptom Inventory. Pain. 2004;108:248-57.

7. Haanpaa M, Attal N, Backonja M, Baron R, Bennett M, Bouhassira D, et al. NeuPSIG guidelines on neuropathic pain assessment. Pain. 2011;152:14-27.

8. Min K, Oh Y, Lee SH, Ryu JS. Symptom-based treatment of neuropathic pain in spinal cord-injured patients: a randomized crossover clinical trial. Am J Phys Med Rehabil. 2016;95:330-8.

9. Freeman R, Baron R, Bouhassira D, Cabrera J, Emir B. Sensory profiles of patients with neuropathic pain based on the neuropathic pain symptoms and signs. Pain. 2014;155:367-76.

10. Padua L, Briani C, Jann S, Nobile-Orazio E, Pazzaglia C, Morini A, et al. Validation of the Italian version of the Neuropathic Pain Symptom Inventory in peripheral nervous system diseases. Neurol Sci. 2009;30:99-106.

11. Sommer C, Richter H, Rogausch JP, Frettloh J, Lungenhausen M, Maier C. A modified score to identify and discriminate neuropathic pain: a study on the German version of the Neuropathic Pain Symptom Inventory (NPSI). BMC Neurol. 2011;11:104.

12. de Andrade DC, Ferreira KA, Nishimura CM, Yeng LT, Batista $\mathrm{AF}$, de Sa K, et al. Psychometric validation of the Portuguese version of the Neuropathic Pain Symptoms Inventory. Health Qual Life Outcomes. 2011;9:107.

13. Villoria J, Rodriguez M, Berro MJ, Stern A, Sanchez-Magro I. Psychometric validation of the Neuropathic Pain Symptom Inventory for its use in Spanish. J Pain Symptom Manag. 2011;42:134-46.

14. Matsubayashi Y, Takeshita K, Sumitani M, Oshima Y, Tonosu J, Kato S, et al. Psychometric validation of the Japanese version of the Neuropathic Pain Symptom Inventory. PLoS One. 2015;10: e0143350.
15. Lu LC, Chang SY, Liu CY, Tsay SL. Reliability and validity of the Chinese version Neuropathic Pain Symptom Inventory in patients with colorectal cancer. J Formos Med Assoc. 2018;117:1019-26.

16. Nagoshi N, Kaneko S, Fujiyoshi K, Takemitsu M, Yagi M, Iizuka $\mathrm{S}$, et al. Characteristics of neuropathic pain and its relationship with quality of life in 72 patients with spinal cord injury. Spinal Cord 2016;54:656-61.

17. Widerstrom-Noga E, Felix ER, Adcock JP, Escalona M, Tibbett J. Multidimensional neuropathic pain phenotypes after spinal cord injury. J Neurotrauma. 2016;33:482-92.

18. Siddall PJ, McClelland JM, Rutkowski SB, Cousins MJ. A longitudinal study of the prevalence and characteristics of pain in the first 5 years following spinal cord injury. Pain. 2003;103:249-57.

19. Safikhani S, Gries KS, Trudeau JJ, Reasner D, Rudell K, Coons SJ, et al. Response scale selection in adult pain measures: results from a literature review. J Patient Rep Outcomes. 2017;2:40.

20. Dworkin RH, Turk DC, Farrar JT, Haythornthwaite JA, Jensen MP, Katz NP, et al. Core outcome measures for chronic pain clinical trials: IMMPACT recommendations. Pain. 2005;113:9-19.

21. Cook KF, Dunn W, Griffith JW, Morrison MT, Tanquary J, Sabata D, et al. Pain assessment using the NIH toolbox. Neurology. 2013;80:S49-53.

22. Kerns RD, Turk DC, Rudy TE. The West Haven-Yale Multidimensional Pain Inventory (WHYMPI). Pain. 1985;23:345-56.

23. Widerstrom-Noga EG, Cruz-Almeida Y, Martinez-Arizala A, Turk DC. Internal consistency, stability, and validity of the spinal cord injury version of the Multidimensional Pain Inventory. Arch Phys Med Rehabil. 2006;87:516-23.

24. Widerstrom-Noga EG, Duncan R, Felipe-Cuervo E, Turk DC. Assessment of the impact of pain and impairments associated with spinal cord injuries. Arch Phys Med Rehabil. 2002;83:395-404.

25. Craig A, Guest R, Tran Y, Nicholson Perry K, Middleton J. Pain catastrophizing and negative mood states after spinal cord injury: transitioning from inpatient rehabilitation into the community. J Pain. 2017;18:800-10.

26. Lazaridou A, Kim J, Cahalan CM, Loggia ML, Franceschelli O, Berna C, et al. Effects of cognitive-behavioral therapy (CBT) on brain connectivity supporting catastrophizing in fibromyalgia. Clin J Pain. 2017;33:215-21.

27. Rosenstiel AK, Keefe FJ. The use of coping strategies in chronic low back pain patients: relationship to patient characteristics and current adjustment. Pain. 1983;17:33-44.

28. Raichle KA, Hanley M, Jensen MP, Cardenas DD. Cognitions, coping, and social environment predict adjustment to pain in spinal cord injury. J Pain. 2007;8:718-29.

29. Attal N, Fermanian C, Fermanian J, Lanteri-Minet M, Alchaar H, Bouhassira D. Neuropathic pain: are there distinct subtypes depending on the aetiology or anatomical lesion? Pain. 2008;138:343-53.

30. Cruz-Almeida Y, Martinez-Arizala A, Widerstrom-Noga EG. Chronicity of pain associated with spinal cord injury: a longitudinal analysis. J Rehabil Res Dev. 2005;42:585-94.

31. Crawford B, Bouhassira D, Wong A, Dukes E. Conceptual adequacy of the Neuropathic Pain Symptom Inventory in six countries. Health Qual Life Outcomes. 2008;6:62. 